\title{
A guide to the removal of heavy silicone oil
}

T Stappler, R Williams, S K Gibran, et al.

Br J Ophthalmol 2008 92: 844-847 originally published online May 6, 2008 doi: 10.1136/bjo.2007.134569

Updated information and services can be found at:

http://bjo.bmj.com/content/92/6/844.full.html

These include:

References This article cites 4 articles, 1 of which can be accessed free at: http://bjo.bmj.com/content/92/6/844.full.html\#ref-list-1

Email alerting Receive free email alerts when new articles cite this article. Sign up in the service box at the top right corner of the online article.

Notes

To request permissions go to:

http://group.bmj.com/group/rights-licensing/permissions

To order reprints go to:

http://journals.bmj.com/cgi/reprintform

To subscribe to BMJ go to:

http://journals.bmj.com/cgi/ep 


\title{
A guide to the removal of heavy silicone oil
}

\author{
T Stappler, ${ }^{1}$ R Williams, ${ }^{2}$ S K Gibran, ${ }^{1}$ E Liazos, ${ }^{1}$ D Wong ${ }^{1,3}$
}

'St Paul's Eye Unit, Royal Liverpool University Hospital, Liverpool, UK; ${ }^{2}$ Clinical Engineering and Ophthalmology, School of Clinical Sciences, University of Liverpool, Liverpool, UK; ${ }^{3}$ Eye Institute, LKS Faculty of Medicine, The University of Hong Kong, Hong Kong, China

Correspondence to: Dr T Stappler, St Paul's Eye Unit, Royal Liverpool University Hospital, Prescot Street, Liverpool L7 8XP, UK; theodor. stappler@rlbuht.nhs.uk

Accepted 10 March 2008

\section{ABSTRACT}

Aim: Heavy silicone oil removal can be challenging and differs considerably from conventional oil. Traditionally, strong active aspiration had to be applied through a long $18 \mathrm{G}$ needle just above the optic disc. We present a novel technique using a much shorter $(7.5 \mathrm{~mm})$ and smaller (20G) needle allowing its removal "from a distance."

Method: Active aspiration on a vacuum of $600 \mathrm{~mm} \mathrm{Hg}$ of the "viscous fluid injector" was applied using the $20 \mathrm{G}$ cannula in a polymethylmethacrylate model eye chamber that was surface-modified to mimic the surface properties of the retina. Measurements were taken using still photographs.

Results: Under injection the maximum diameter of a silicone oil bubble supported by interfacial tension alone was $5 \mathrm{~mm}$ for a steel and $7 \mathrm{~mm}$ for a polyurethane cannula. Under suction, the silicone bubble changed shape and became conical, thus further increasing the cannula's reach. This conical shape illustrated "tubeless siphoning," which is a physical property of non-Newtonian fluids.

Discussion: The use of shorter and smaller gauge cannula for removal of Densiron obviates the need to enlarge the sclerotomy beyond $20 \mathrm{G}$ or to apply suction in close proximity to disc and fovea. This potentially reduces the risk of iatrogenic damage such as entry site tears or postoperative hypotony.

The use of Densiron heavy silicone oil has been piloted by our group and has shown to be an effective tamponade agent in a two-centred pilot study involving Rotterdam and Liverpool. ${ }^{1}$ Densiron, being heavier than water, presents special problems during its removal. While there is no limit to the pressure that can be applied for injection of a viscous fluid, when it comes to aspiration, the maximum suction that can be generated (even if a complete vacuum were to be applied) is one atmosphere. For this reason, large bore needles had to be used; otherwise the flow would be too slow. Additionally, the tip of the needle needs to reach the lowest part of the vitreous cavity, or so it was thought (fig 1A).

This conventional way of removing Densiron has several drawbacks. For a 20- or smaller gauge vitrectomy approach, one of the sclerotomies needs to be enlarged. Potentially there is an increased risk of complications relating to the larger scleral openings including entry site tears. There might also be risks associated with applying high suction pressure close to the disc and fovea. If the needle was momentarily emptied of the viscous oil, there would be a sudden increase in flow, and the needle tip could damage the retina as the eye suddenly collapsed with hypotony.

Empirically, we observed that it was possible to remove heavy oil using a 20 -gauge cannula of only
$7.5 \mathrm{~mm}$ in length using $600 \mathrm{~mm} \mathrm{Hg}$ of suction generated by the syringe driver (fig 1B). It was therefore possible to avoid many of the abovementioned problems by removing the heavy oil "from a distance." This has been our routine even in eyes with axial myopia.

The aim of this study is to see if we could illustrate and thus better understand the forces involved when we remove Densiron using a short cannula.

We considered the main forces involved. The downward force is the gravity acting on the bubble. There is also adhesion between Densiron and the retina: the retina is relatively hydrophilic, and Densiron is hydrophobic; the interfacial energy between Densiron and the retina is therefore low, but nonetheless there exists an attractive force acting to resist the aspiration of the bubble of Densiron. The upward forces include the buoyancy of the bubble. There is the adhesion generated by the interfacial energy between the Densiron and the cannula. This adhesion means that the cannula can suspend a droplet of Densiron against the force of gravity. The magnitude of this adhesion varies depending on the surface property of the cannula, be it made of metal or plastic.

There may be an additional force that is derived from the extensional strain caused by the suction of the syringe. This force is responsible for the phenomenon described as "tubeless siphoning." We are familiar with normal siphoning, which allows fluid to drain from a reservoir with fluid flowing upwards driven by hydrostatic pressure before flowing down through a tube (fig 2A). The idea of a tubeless siphon is that some viscoelastic fluid can flow upwards even without a tube, driven by suction and by the stored energy of extensional flow (fig 2B). If Densiron could be removed using a short cannula in vivo, then we should be able to demonstrate this tubeless siphon in vitro.

The aim of this study is to see if we could illustrate and thus better understand the forces involved when we remove Densiron using a short cannula.

\section{METHOD}

\section{Materials}

Densiron has a viscosity of $1480 \mathrm{mPas}$ and a specific gravity of $1.06 \mathrm{~g} / \mathrm{ml}$. We used a model eye chamber made of PMMA previously described by us in other studies; it had an internal diameter of $23 \mathrm{~mm}$ and had been surface-modified to mimic the hydrophilic surface properties of the retina. ${ }^{2}$ Only the lower half of the model eye chamber was used, as the upper half did not contribute to the support of the Densiron bubble. We used two types of cannulae: a 20-gauge metal cannula (Viscous Fluid Injector, Accurus (Alcon)) and a 
Figure 1 (A) Removal of heavy oil: conventional method. (B) Removal of heavy oil: novel method.
A

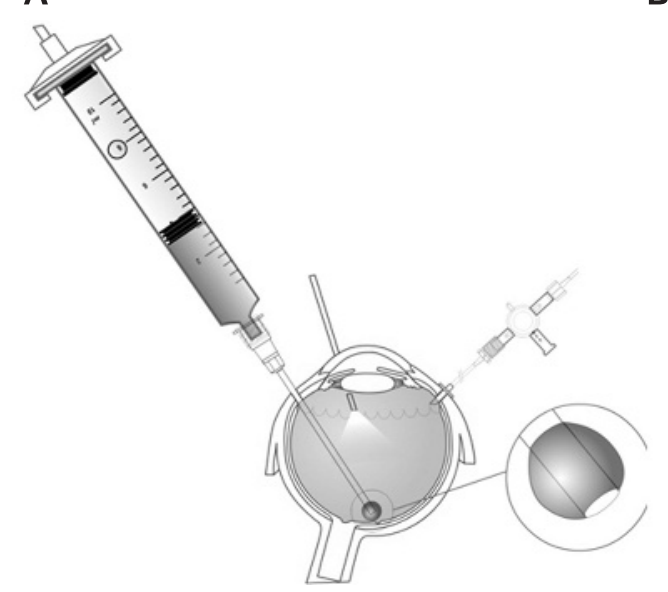

B

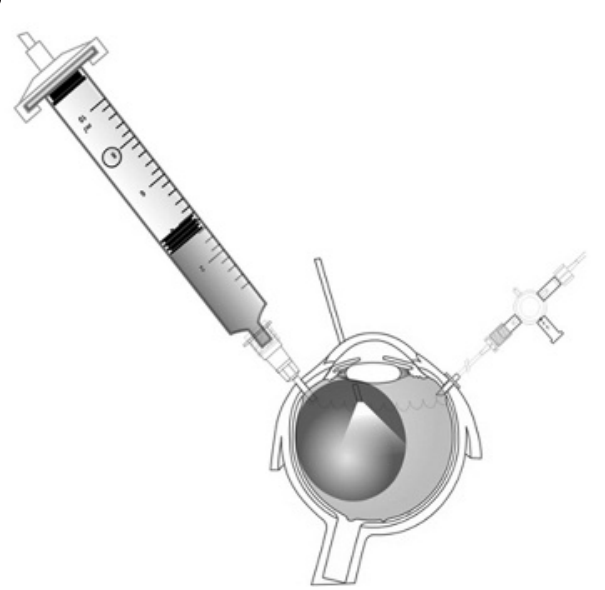

20-gauge polyurethane cannula (Venflon). The metal cannula was approximately $7.5 \mathrm{~mm}$ in length from the plastic hub to the tip of the cannula. The Venflon cannula was cut to the same length. We recorded the shape and the dimension of the Densiron using photography. Photographs were taken during injection as well as aspiration of Densiron.

\section{Aspiration}

Aspiration was generated by the Accurus (Alcon) vitrectomy machine that had a maximum suction of $600 \mathrm{~mm} \mathrm{Hg}$. This suction was applied via a syringe driver called the "Viscous Fluid Injector" (Alcon), and the aspiration needles were connected directly to the syringe.

\section{Photography}

Digital photographs were taken during injection and aspiration using a digital camera. The "macro" setting of the camera was used to obtain a magnified view.

\section{Injection}

Densiron from a syringe driver was injected into a balanced salt solution using both needles. A series of still photographs were taken to capture the maximum size of the droplet before it broke off from the cannula; first the metal, then the polyurethane cannula was used.
A

\section{Normal siphon}

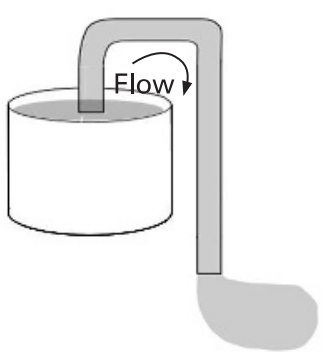

B

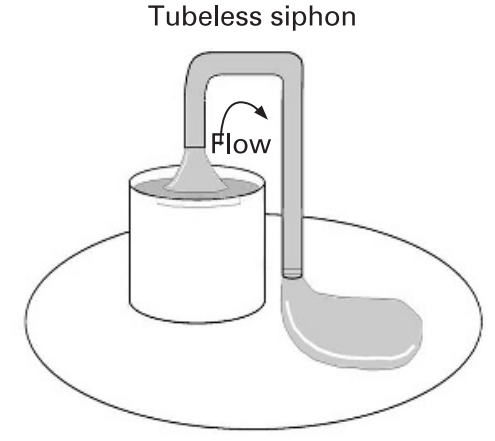

Figure 2 (A) Regular siphon. (B) Tubeless siphon.

\section{Aspiration}

A large bubble of Densiron was placed within the model eye chamber and aspirated; its progress was monitored with a series of photographs using the two cannulae.

\section{RESULTS}

During injection, the adhesion of Densiron to the cannula was able to suspend a bubble without the bubble breaking off and falling away from the cannula. In the case of the metal cannula, the bubble reached a size of $5 \mathrm{~mm}$ in diameter before the oil droplet became detached and fell down. With the polyurethane cannula, the bubble reached $7 \mathrm{~mm}$ in diameter. The injections gave an indication of the maximum volume of a droplet that could be supported by interfacial tension alone between the above materials.

During aspiration, we observed that the bubble sitting on the model eye chamber was drawn into a cone shape (fig $3 \mathrm{~A}$ ). This was a manifestation of the phenomenon of the tubeless siphon. It was as though the aspiration was drawing up a peak or a tube from the main oil droplet into the aspirating needle creating an even more pronounced conical shape (fig 3B). This further increased the effective "reach" of the short cannula. It also demonstrated the cohesiveness of Densiron under suction. The cohesiveness refers to the tendency of the liquid to stay as a single bubble rather than breaking into two or more elements.

The in vitro experiment, however, did not succeed in removing oil with the tip of the needle more than about $10 \mathrm{~mm}$ from the bottom of the eye model chamber. When a short metal cannula (that comes as part of the "viscous fluid injector") is inserted into the eye via the pars plana, the tip of a metal cannula would be still about $15 \mathrm{~mm}$ from the optic disc, depending on the thickness of the sclera at the sclerotomy and
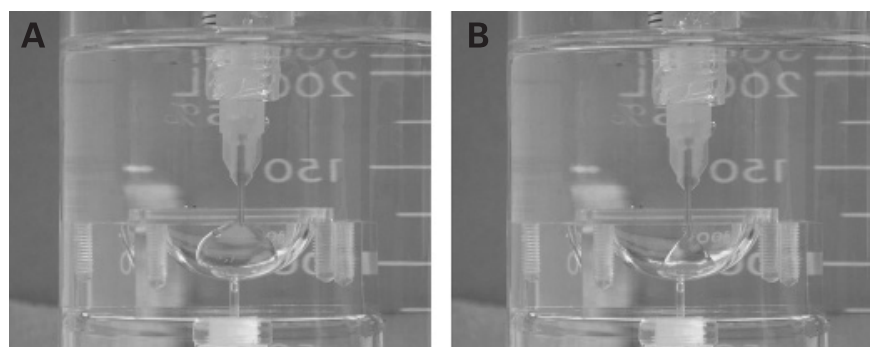

Figure 3 Tubeless siphoning. Suction distorts the shape of the Densiron bubble, therefore increasing its reach even further. 
the axial length of the eye. Clinically, though, we are consistently able to remove Densiron using the $7.5 \mathrm{~mm}, 20$ gauge cannula from both normal and short-sighted eyes.

\section{DISCUSSION}

When aspirating any viscous fluid, consideration has to be given to Poiseuille's Law. Any aspiration via long tubing would not be effective. The drop in aspiration pressure along the length of the tubing would mean that the suction at the distal end of the tubing would be too low to be sufficient to suck out the viscous fluid. For this reason, a syringe driver needed to be used. This meant that the suction was generated at the hub of the needle, and the only length involved was the length of the needle itself. Equally, the flow critically depended on the 4th power of the diameter of aspiration needle. For this reason, even though the aspirating needles were only $25-30 \mathrm{~mm}$ in length, they needed to be 19- or even 18-gauge to have sufficient flow. Such long needles were used because it was thought that the needle tip needed to reach the optic disc of the eye. If it were possible to aspirate Densiron by employing the phenomenon of tubeless siphon, it would be possible to use shorter and thus smaller bore needles ( $7.5 \mathrm{~mm}$ long 20 -gauge). The use of the short needle was also advocated by Wolf et al with reference to the removal of the much more viscous, but less heavy Oxane HD (3000 mPas and $1.02 \mathrm{~g} / \mathrm{ml}) .^{3}$

Poiseuille's law applies to Newtonian fluids. Silicone and Densiron are made of polymers and are viscoelastic. They behave as non-Newtonian fluids. When such non-Newtonian fluids are forced through a narrowing opening, the threedimensional structure of the molecules unfolds, thereby storing up energy in the process. It is this stored energy that is responsible for the "tubeless siphon." ${ }^{5}$ In other words, the liquid can be drawn up towards the suction as though virtually it is being aspirated via a much longer tube or cannula. The fluid would typically have a conical shape, and this is indeed what we have observed in vitro (fig $3 \mathrm{~A}, \mathrm{~B}$ ). In the model eye chamber, we can see the cone-shaped and the "tubeless siphon" because we are observing the bubble from the side, whereas peroperatively, we are looking at the bubble from the top and along the needle (fig 4).

The in vitro experiment demonstrated the surface energy that enables the metal or polyurethane cannula to hold on to a bubble of Densiron, thereby increasing the "reach" of the needle. Dresp and Menz ${ }^{6}$ also showed that the addition of surfactants to the aqueous environment allows longer contact between the oil and the needle. There are a couple of practical

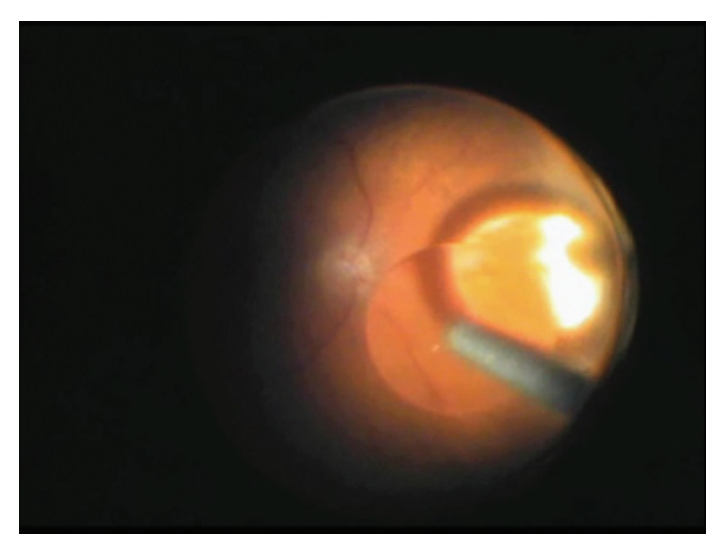

Figure 4 Heavy silicone oil bubble levitated by the short 20G cannula in the middle of the vitreous cavity. points to be considered. The advantage of the Venflon polyurethane cannula is that we can cut it to the desired length. Because Densiron is more adherent to it, there is often a film of oil on the outside the cannula which cannot be easily removed. The film is "scraped off" the cannula when it is removed from the eye. This can leave a droplet of the Densiron behind. These droplets are of course heavier than water and can annoy patients and disturb their vision when they lie down. A second more important practical consideration is that the use of a short cannula to remove Densiron is reliant on tubeless siphoning. Like any siphoning, if the fluid was interrupted, it would not be possible to pick up the bubble again and resume flow. The surgeon must be careful to make sure that the needle stays inside the bubble of Densiron to be removed at all times.

In the experiment, it was not possible to remove a large bubble of Densiron with the tip of the needle more than $10 \mathrm{~mm}$ away from the lowest part of the model eye cavity. There may be three important differences between the model chamber and the human eye. First, it has been pointed out that the maximum suction that can be generated by a vacuum is one atmosphere. That is the case when the aspiration is acting in a chamber opened to the atmosphere. One could think of it as the atmospheric pressure that is "pushing" the fluid into the vacuum of the syringe driver. The eye is a closed chamber. By having an infusion into the eye and by raising the infusion bottle, the pressure differential can be increased. Accordingly, the tubeless siphoning effect will be increased. We recommend raising the infusion bottle when removing Densiron in myopic eyes with a high axial length. The height of the infusion bottle and the duration of sustained raised intraocular pressure should of course be compatible with the safe perfusion of the eye.

Second, the model chamber did not take into account any effects of intraocular currents, which may further lift the bubble of oil from the retina. Lastly, the albumin solution modified the surface of the chamber and made it hydrophilic. It is known that proteins in the aqueous can also act as surfactants, thus further reducing the surface energy of the Densiron bubble. The adhesion between the Densiron and the retina could therefore be less than that between the oil and the chamber.

There have been reports of sticky Oxane HD that made its removal more difficult. ${ }^{3}$ Once a film of Oxane HD was left on the retina, long needles would be required to be used to aspirate the liquid close to retinal surface. The aspiration pressure at the tip of a long needle would be low once they were completely filled with the high-viscosity oil. The inability of long needles to remove Oxane HD may be due to a genuine "stickiness" causing the heavy oil to adhere to the retina. Equally, it might be that the suction via a long needle would be low if the needle were filled with oil. Dresp and Menz postulated that the adhesion might be due to trace elements of perfluorocarbon liquid. ${ }^{6}$ There are also anecdotal reports of sticky PFCL, conventional silicone oil and Densiron. As a result of our experiments, we cannot offer advice as to the best way of removing thin films of these tamponade agents.

In summary, a combination of static forces such as the interfacial tension between the steel or plastic cannula and the oil as well as dynamic forces such as the phenomenon of tubeless siphoning made it feasible to use shorter and smallergauge needles to remove heavy silicone oil. We recommend the use of the short 20-gauge steel needle because it works, and it reduces the risk of iatrogenic damage.

Competing interests: None 


\section{REFERENCES}

1. Wong D, Van Meurs J, Stappler T, et al. A pilot study on the use of a perfluorohexyloctane/silicone oil solution as heavier than water internal tamponade agent. Br J Ophthalmol 2004;88:692-6.

2. Fawcett IM, Williams RL, Wong D. Contact angles of substances used for internal tamponade in retinal detachment surgery. Graefes Arch Clin Exp Ophthalmol 1994;232:438-44.
3. Wolf S, Schon V, Meier P, et al. Silicone oil-RMN3 mixture ("heavy silicone oil") as internal tamponade for complicated retinal detachment. Retina 2003;23:355-42.

4. Bird RB, Armstrong RC, Hassager 0. Dynamics of polymeric liquids. Volume 1: fluid mechanics, 2nd edn. New York: Wiley Interscience, 1987:75.

5. Boger DV, Walters K. Rheological phenomena in focus. Amsterdam: Elsevier, 1993:29.

6. Dresp JH, Menz DH. The phenomenon of "sticky" silicone oil. Graefes Arch Clin Exp Ophthalmol 2007;245:863-8. 